\title{
Absence of Large-Scale Dendritic Plasticity of Layer 5 Pyramidal Neurons in Peri-Infarct Cortex
}

\author{
Ricardo Mostany and Carlos Portera-Cailliau \\ Departments of Neurology and Neurobiology, David Geffen School of Medicine, University of California, Los Angeles, Reed Neurological Research Center, \\ Los Angeles, California 90095
}

When stroke or traumatic brain injury lead to cortical damage, how do surviving neurons rewire the brain to restore lost functionalities? Several Golgi studies have argued for de novo growth and branching of dendrites of pyramidal neurons in the spared hemisphere, but the results could not always be replicated. Functional brain imaging studies in humans and rodents suggest that significant neuronal plasticity occurs in areas surrounding the cortical lesion, but whether dendritic rearrangements occur there has been less well studied, especially after stroke. We used in vivo two-photon microscopy in adult mice expressing green fluorescent protein to monitor longitudinally the length and branch complexity of entire apical dendritic arbors from layer 5 pyramidal neurons distributed over a large peri-infarct cortex region after middle cerebral artery occlusion. We find no evidence of growth of dendrites or addition of new branches to their arbors over a period of 3 months after stroke. Instead, we observed a two-step pruning process: an initial decrease in dendritic length, followed by a loss of dendritic branches. Importantly, the shortening of branch tips reflected a general shrinkage in the dendritic apical tree, suggesting that mechanical forces attributable to the involution of the infarct contributed to the changes in dendritic length. These results help resolve a long-standing debate regarding the role of large-scale dendritic plasticity of pyramidal neurons in functional recovery after cortical injury.

\section{Introduction}

Many of the sensory, motor, and cognitive impairments caused by stroke eventually improve, suggesting that the brain has the ability to rewire itself to restore lost functionalities (Carmichael, 2008). The modification of neuronal connections may result from axonal sprouting, neurogenesis, or remodeling of dendritic arbors (Zhang and Chopp, 2009). A better understanding of neuronal plasticity in functional recovery after stroke will be crucial to the development of pharmacologic and rehabilitative therapies for both ischemic and traumatic brain injury.

Golgi studies have suggested that de novo growth and branching of pyramidal neuron dendrites in the spared hemisphere play a role in circuit plasticity after cortical injury (Jones and Schallert, 1992). However, the evidence is controversial, because other studies could not replicate those results (Forgie et al., 1996; Prusky and Whishaw, 1996) or found instead a shortening of dendrites after cortical lesions, including stroke (Biernaskie et al., 2004; Gibb et al., 2010).

Much less is known about dendritic remodeling after cortical injury in neurons immediately surrounding the cortical lesion,

\footnotetext{
Received Aug. 20, 2010; accepted Nov. 24, 2010.

This work was supported by grants from the Larry L. Hillblom Foundation and The March of Dimes Foundation. We thank Abinav Baweja for technical assistance and Dr. S. Thomas Carmichael for guidance at early stages of this project.

Correspondence should be addressed to either Ricardo Mostany or Carlos Portera-Cailliau, Departments of Neurology and Neurobiology, David Geffen School of Medicine, University of California, Los Angeles, Reed Neurological Research Center, Room A-145, 710 Westwood Plaza, Los Angeles, CA 90095. E-mail: mostany@ucla.edu, cpcailliau@mednet.ucla.edu.

DOI:10.1523/JNEUROSCI.4386-10.2011

Copyright $\odot 2011$ the authors $\quad 0270-6474 / 11 / 311734-05 \$ 15.00 / 0$
}

although functional imaging studies in humans and rodents point to significant remapping taking place in that region (Marshall et al., 2000; Dijkhuizen et al., 2001). One such study that examined dendritic length and branching of pyramidal neurons in peri-infarct cortex after middle cerebral artery occlusion (MCAO) found no differences between lesioned and control animals (Gonzalez and Kolb, 2003).

A shortcoming of Golgi studies is that dynamic aspects of largescale dendritic remodeling could not be examined. In this regard, a recent longitudinal in vivo imaging study found evidence for balanced elongation-retraction of pyramidal dendrites as a mechanism of large-scale dendritic plasticity in peri-infarct cortex after a small photo-thrombotic stroke (Brown et al., 2010).

Still, the notion that dendrites would grow or add new branches to compensate for lost functionalities after cortical injury is at odds with the remarkable stability of dendritic arbor size that in vivo imaging has demonstrated for cortical pyramidal cells in the normal adult brain (Trachtenberg et al., 2002; Lee et al., 2006). Thus, whether large-scale dendritic plasticity occurs in pyramidal neurons after stroke, especially in peri-infarct cortex, remains an unresolved issue. Settling this debate is important because layer 5 (L5) pyramidal neurons are the sole excitatory output of the cortex, and significant changes in the size of dendritic arbors could have a major impact on the cortical circuit.

Here, we examined the entire apical dendritic arbor of 11 L5 pyramidal cells in peri-infarct cortex across multiple imaging sessions over 3 months using in vivo two-photon microscopy in the clinically relevant MCAO stroke model. Careful reconstructions of these arbors did not reveal an increase in dendritic complexity in L5 neurons and showed instead a slight pruning of dendritic branches. 
a
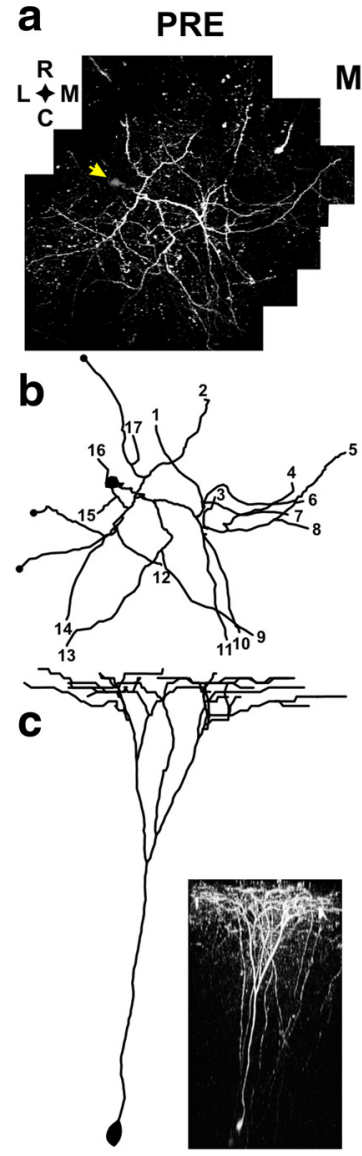

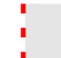

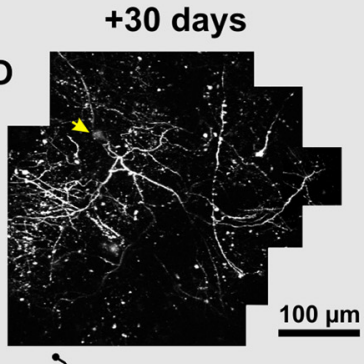

$$
+30 \text { days }
$$

MCAO :

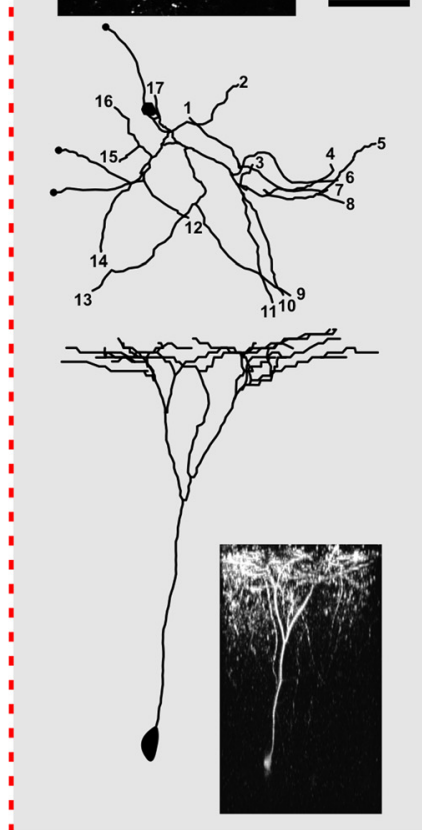

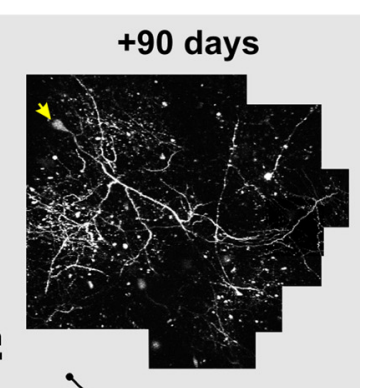
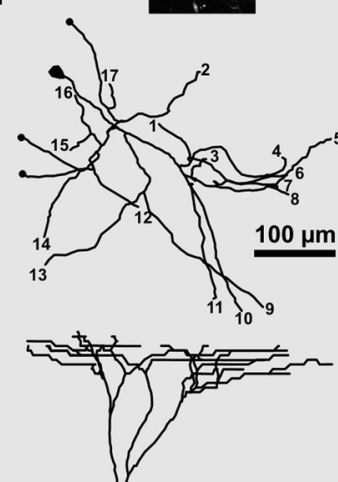

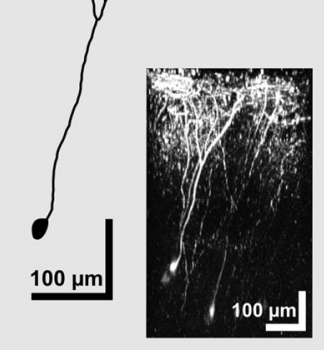

Figure 1. Long-term in vivo two-photon imaging of $L 5$ pyramidal cells in peri-infarct cortex in GFP-M mice. $\boldsymbol{a}$, Maximum intensity projections ( $x y$; 120 slices, $5 \mu \mathrm{m}$ apart) of a representative $L 5$ pyramidal neuron imaged in vivo before (PRE, left) and after ( +30 and +90 d, middle and right, respectively, shaded area) permanent unilateral MCA0. Yellow arrows, Cell body; $R$, rostral; $C$, caudal; L, lateral; M, medial. $\boldsymbol{b}$, Top view of a three-dimensional reconstruction of the same cell. $\boldsymbol{c}$, Side view of the reconstruction. Insets, Side projections $(x z)$ of the image stacks shown in $\boldsymbol{a}$.

\section{Materials and Methods}

Animals. We used a total of nine adult (3-6 months of age; male and female) GFP-M transgenic mice (green fluorescent protein mice, line M) (Feng et al., 2000); six were subjected to stroke, and three served as controls. All procedures were approved by the University of California Chancellor's Animal Research Committee.

Cranial window surgery. We implanted chronic glass-covered cranial windows as described previously (Mostany and Portera-Cailliau, 2008; Holtmaat et al., 2009). A craniotomy was performed using a dental drill $3 \mathrm{~mm}$ lateral to the midline and $1.95 \mathrm{~mm}$ caudal to bregma. A sterile glass coverslip was placed over the dura mater and glued to the skull with cyanoacrylate glue. Dental acrylic was then applied throughout the exposed skull surface around the edges of the coverslip. To later secure the mouse onto the microscope stage, a small titanium bar was embedded in the dental acrylic. Mice were allowed to recover from the surgery for at least 3 weeks before the first imaging session.

Distal middle cerebral artery occlusion. A unilateral and permanent distal MCAO was performed after the baseline imaging session as described previously (Tamura et al., 1981; Mostany et al., 2010). The MCA was occluded with the tip of a small forgery iron through a small craniotomy. Control animals underwent the same procedure, but the MCA was not cauterized.

In vivo two-photon imaging of apical dendritic arbors from L5 pyramidal cells. We used a custom-built two-photon microscope, using a titanium: sapphire laser (Chameleon Ultra II; Coherent) tuned to $910 \mathrm{~nm}$, a $40 \times, 0.8$ numerical aperture water-immersion objective (Olympus), and ScanImage software (Pologruto et al., 2003) written in MATLAB (MathWorks). The spatial resolution of our system is $0.49 \pm 0.18 \mu \mathrm{m}$ for the $x$ - $y$-axis and
$3.65 \pm 0.35 \mu \mathrm{m}$ for the $z$-axis (mean $\pm \mathrm{SD}, n=$ 10 beads) (supplemental Fig. $1 a$, available at www.jneurosci.org as supplemental material). We imaged all mice anesthetized with isoflurane $(\sim 1.2 \%)$ before MCAO (PRE) and over multiple sessions after the real/mock MCAO procedure. We imaged the entire apical dendritic tree (from dura to cell body) of one or two L5 pyramidal neurons per animal at lowmagnification $(512 \times 512$ pixels, $0.72 \mu \mathrm{m} /$ pixel, $5 \mu \mathrm{m} z$ steps, $110-150$ optical slices per stack). At $+8,+12$, +16 , and $+20 \mathrm{~d}$, we imaged the apical dendritic tufts only (from the point where the apical dendrite first branches all the way to the dura; ranging from 185 to 450 $\mu \mathrm{m}$ deep). We obtained higher-magnification images $(512 \times 512$ pixels, $0.152 \mu \mathrm{m} /$ pixel, 1.5 $\mu \mathrm{m} z$ steps, $5-12$ slices per stack) from selected dendritic tips from the same neurons at more frequent intervals for closer inspection of growth/pruning of dendritic tips. Specifically, we imaged and analyzed the lengths of dendritic tips twice before the MCAO (at $-8 \mathrm{~d}$ and at day 0 , right before MCAO) and again at +4 , $+8,+16,+30$, and $+90 \mathrm{~d}$ after MCAO. All imaged tips were in $\mathrm{L} 1$ (within $100 \mu \mathrm{m}$ below the dura), and we focused on dendrites that coursed parallel to the imaging plane.

Analysis. Neurons were reconstructed three dimensionally from image stacks using Neurolucida (MBF Bioscience). All imaged apical dendrites were included in the analysis of branching complexity; apical dendrite tips were included in the analysis of dendritic terminal lengths only if they could be identified unequivocally at every single imaging session. Neurolucida reconstructions were then exported to NeuroExplorer for the analysis of apical dendritic tree complexity and length. Sholl analysis was performed with a concentric radial interval of $10 \mu \mathrm{m}$. We reconstructed 11 different L5 pyramidal cells from six mice for the stroke group and five cells from three mice for the control group.

High-magnification images containing dendritic terminals were analyzed using NIH ImageJ software (http://rsbweb.nih.gov/ij/). A total of 43 dendritic tips from 10 cells (stroke group) and 23 tips from 5 cells (control group) were analyzed over time.

We used GraphPad Prism (GraphPad Software) to compute one-way and two-way repeated measures ANOVA, followed by Bonferroni's or Dunnett's multiple comparison tests, as well as Student's $t$ test for pairwise comparison. All data are presented as mean \pm SEM. Significance was set at $p<0.05$.

\section{Results}

\section{Reduced L5 pyramidal cell dendritic complexity in} peri-infarct cortex after MCAO

To investigate whether large-scale dendritic plasticity of pyramidal neurons occurs after stroke, we repeatedly imaged L5 neurons ( $n=$ 11) in vivo through cranial windows implanted in Thy1-GFP mice before and after permanent unilateral MCAO (Fig. 1). We focused on L5 neurons located in peri-infarct somatosensory cortex (average of $1.9 \mathrm{~mm}$ away from the infarct edge; range, $1.30-2.32 \mathrm{~mm}$ ) that survived after stroke. The permanent MCAO produced large infarcts involving the primary somatosensory cortex, with diameters of $3.22 \pm 0.7 \mathrm{~mm}$ in the mediolateral axis.

The loss of brain tissue associated with stroke after MCAO was accompanied by a distortion of the cortex surrounding the infarct. This was evident in the morphology of L5 cells after the 
a
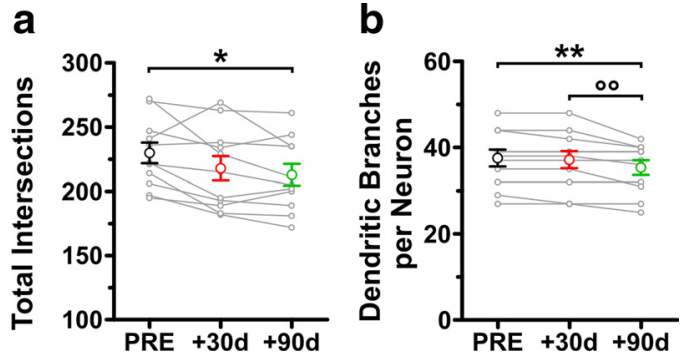

Figure 2. Decreases in apical dendritic complexity after stroke. $\boldsymbol{a}$, Total number of intersections of the $\mathrm{L} 5$ apical dendritic tree before and after $\mathrm{MCAO}$, as determined by three-dimensional Sholl analysis. ${ }^{*} p<0.05$ PRE versus $+90 \mathrm{~d}$. $\boldsymbol{b}$, Number of apical dendritic branches per cell. ${ }^{* *} p<0.01$, PRE versus $+90 \mathrm{~d} ;{ }^{\circ} p<0.01,+30$ versus $+90 \mathrm{~d}$.

stroke, which exhibited bending and stretching of dendrites (Fig. 1) (supplemental Fig. 2, available at www.jneurosci.org as supplemental material). Despite these distortions, apical dendritic trees could be easily resolved for three-dimensional reconstructions (supplemental Movies 1-3, available at www.jneurosci.org as supplemental material) to measure lengths of dendritic segments repeatedly over several weeks, except when the tips extended beyond the image stack, when they were obscured by blood vessels, or when the density of GFP-expressing neurons was too high to reliably trace dendrites (Fig. $1 b, c$ ).

Scholl analysis of apical dendritic branches revealed a decrease in the number of intersections after stroke $\left(_{(2,32)}=5.83, p=\right.$ $0.0101 ; 7.7 \pm 2.1 \%$ lower at $+90 \mathrm{~d}$ vs baseline; post hoc test, $p<0.05$ ) (Fig. 2a). Because this decrease in the number of intersections per cell could be attributable to a reduction in the number of branches and/or to a shortening of the apical dendritic tree (or part of it), we counted the number of dendritic branches per neuron and found it to be significantly reduced at $+90 \mathrm{~d}$ after $\operatorname{MCAO}\left(F_{(2,32)}=9.61, p=0.0012 ;\right.$ post hoc test, $\left.p<0.01\right)$ (Fig. $2 b)$. This reduction in number of branches affects more specifically fifth- and sixth-order branches rather than the more distal (seventh to ninth order) branch tips (supplemental Fig. 3a, available at www.jneurosci.org as supplemental material). We speculate that the reason for this observation is that fifth and sixth branch orders are the orders with the highest number of dendritic tips, which may be more susceptible to pruning. Actually, $70 \%$ of the tips belonging to the cells included in this study before the MCAO belong to the fifth or sixth order (supplemental Fig. $3 b$, available at www.jneurosci.org as supplemental material). Interestingly, the reduction in the number of branches occurs only after $+30 \mathrm{~d}(p<0.001$, PRE, $+8,+16$, and $+30 \mathrm{~d}$ vs $+90 \mathrm{~d}$; data not shown), suggesting that the loss of branches in peri-infarct cortex is a delayed phenomenon. L5 neurons $(n=5)$ from control mice did not show significant differences in dendritic complexity over time (supplemental Fig. 4, available at www. jneurosci.org as supplemental material).

\section{Shortening of apical dendritic arbors of L5 pyramidal cells after MCAO}

The total length of the apical dendritic tree was significantly reduced after the MCAO $\left(F_{(2,29)}=12.97, p=0.0003\right)$ (Fig. 3a). This effect was already apparent at $+30 \mathrm{~d}$ ( $p<0.01 \mathrm{vs}$ PRE), suggesting that dendritic shortening occurs more acutely than the loss of branches. The first- and second-order, as well as ninthorder, branches were the most affected ( $p<0.01$ PRE vs +30 and +90 d) (supplemental Fig. $3 d$, available at www.jneurosci.org as supplemental material). In addition, last-order branches showed a significant reduction in their length at $+90 \mathrm{~d}$ after MCAO
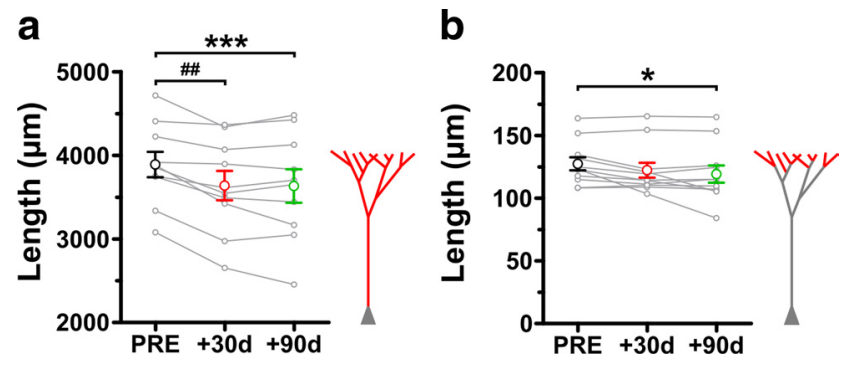

Figure 3. Reduced apical dendritic length of $L 5$ pyramidal cells in peri-infarct cortex. $\boldsymbol{a}$, Total apical dendritic length before and after stroke. ${ }^{\# \#} p<0.01$, PRE versus $+30 \mathrm{~d}$; ${ }^{* * *} p<0.001$, PRE versus $+90 \mathrm{~d}$. $\boldsymbol{b}$, Length of last-order branch tips. ${ }^{*} p<0.05$, PRE versus $+90 \mathrm{~d}$.

$\left(F_{(2,32)}=3.80, p=0.0398\right)$ (Fig. $\left.3 b\right)$. Cells from control animals did not exhibit length changes in their apical dendritic arbor, even in the last-order branches (supplemental Fig. 4, available at www.jneurosci.org as supplemental material).

\section{Pruning at tips and shrinkage of the dendritic shaft account for the shortening of dendritic segments after MCAO}

We observed significant distortions in the dendritic arbors after stroke (Fig. 1), as well as reductions in the thickness of the cortex (supplemental Fig. 2, available at www.jneurosci.org as supplemental material), presumably caused by involution of the neighboring infarcted tissue. Consequently, it is possible that the changes in the length of dendritic tips could be attributable to shrinkage of the entire dendritic tree (Fig. $3 a$ ) rather than to retraction at distal tips (Fig. 3b). The fact that first- and secondorder branches showed the most significant reductions in their lengths (supplemental Fig. $3 d$, available at www.jneurosci.org as supplemental material) implies that a generalized tissue shrinkage may contribute to the shortening of distal dendritic tips. To investigate this, we analyzed changes in length in a subset of dendritic tips $(n=43)$ imaged at high resolution (Fig. 4a). First, we measured the distance from the tip of the dendritic segment to the most distal fiduciary point (e.g., a persistent spine) that could be identified reliably throughout the whole study (Fig. $4 a$, stars) (supplemental Fig. 6, available at www.jneurosci.org as supplemental material). We observed that tips are very stable under basal conditions before the stroke $(+0.33 \pm 0.3 \mu \mathrm{m}$ length change over $8 \mathrm{~d}$ ). However, after the MCAO, there is a cumulative decrease in length of dendritic tips $\left(F_{(5,257)}=25.90, p<0.0001\right.$; post hoc test, $p<0.001$, PRE vs $+8,+16,+30$, and $+90 \mathrm{~d}$ ), even during the first few days after stroke $(-3.9 \pm 0.9 \mu \mathrm{m}$ length change over the first $8 \mathrm{~d} ; t_{(42)}=5.15, p<0.0001$ vs $8 \mathrm{~d}$ baseline) (Fig. $4 b$ ). In contrast to the length of distal dendritic tip segments in control animals, which changed minimally over time, distal tips in stroke animals retracted after MCAO $\left(F_{(1,64)}=12.14, p=\right.$ 0.0009; PRE to $+30 \mathrm{~d}, p<0.01 ;+30$ to $+90 \mathrm{~d}, p<0.05$ ) (Fig. $4 c$ ). Next, we examined whether the changes in total length of the terminal branches (from the last bifurcating point to the end of the segment) differed from the changes in the length of the tips (from the fiduciary point to the tip) of the same dendrite. We found a significant positive correlation between these two parameters (Pearson's $r=0.64, p<0.01$ ) (Fig. $4 d$ ), indicating that the decreases in both were commensurate. However, the slope of the correlation $(0.41 \pm 0.09)$ suggests that the change in the length of a given terminal branch is affected $\sim 40 \%$ by shrinkage/stretching of the entire dendritic shaft and $\sim 60 \%$ by retraction of the terminal tip.

We also examined whether the extent of dendritic pruning might differ between cells located close to versus far away from 
a
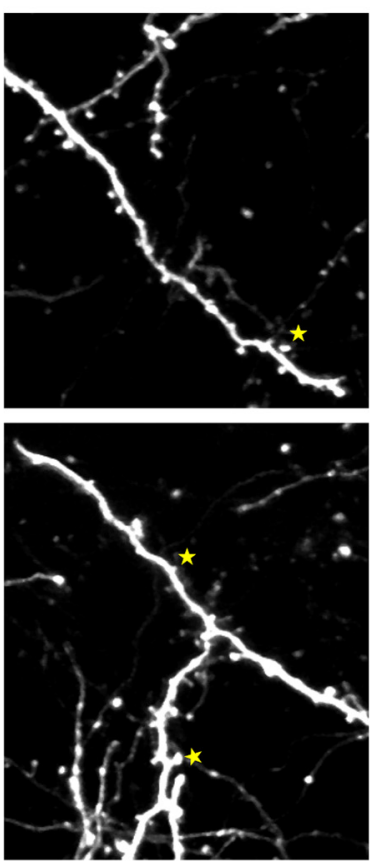

b

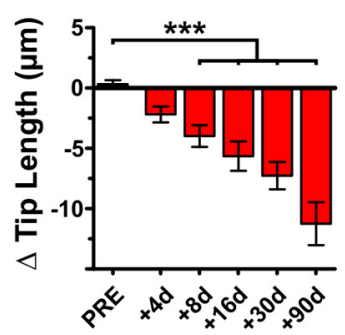

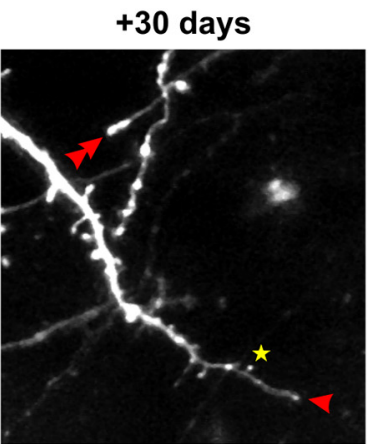

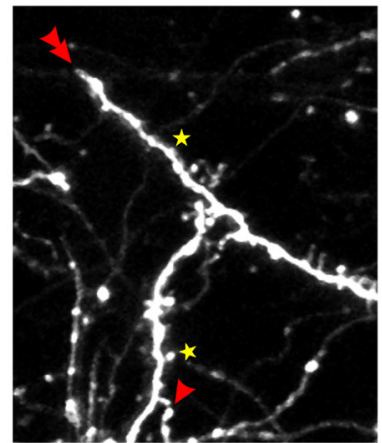

C

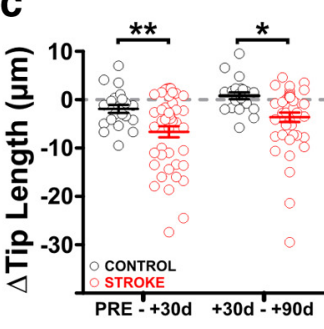

d
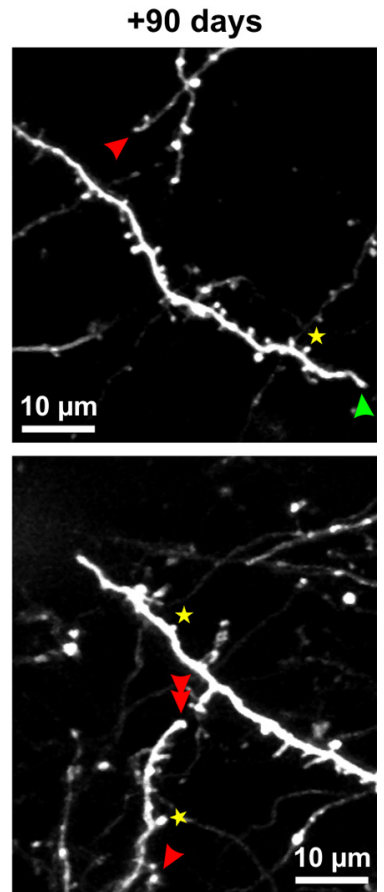

$\triangle$ Branch Length $(\mu \mathrm{m})$

$\begin{array}{lllllll}-40 & -30 & -20 & -10 & 0 & 10 & 20\end{array}$

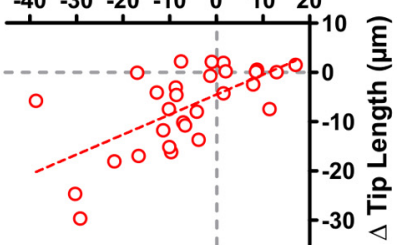

Figure 4. Dendritic shortening is attributable to both retraction of tips and shrinkage/distortion of dendritic shafts. $\boldsymbol{a}$, Representative examples of changes in dendritic tip length. Images are maximum intensity projections $(x y)$ of $10-12$ focal planes, 1.5 $\mu \mathrm{m}$ apart. Yellow stars, Stable fiduciary points used for tip measurements. Single and double red arrowheads represent minimal and moderate dendritic tip retractions, respectively. Green arrowheads represent slight growth. $\boldsymbol{b}$, Change in dendritic tip length before (PRE, baseline over an $8 \mathrm{~d}$ period) and at $+4,+8,+16,+30$, and $+90 \mathrm{~d}$ after MCAO (with respect to the time point right before the MCAO). ${ }^{* * *} p<0.001$. c, Change in dendritic tip length over the first $30 \mathrm{~d}$ after the sham surgery (control) or MCAO (stroke) or between +30 and +90 d. ${ }^{*} p<0.05$ and ${ }^{* *} p<0.01$. Note how shrinkage of dendritic tips is an ongoing phenomenon after stroke, with most of the pruning occurring in the first $30 \mathrm{~d}$ after stroke and how changes in dendritic length after stroke are of larger magnitude than the growth and retraction of dendritic tips observed in control mice. $\boldsymbol{d}$, Significant correlation between the changes in tip length and last-order branch length (Pearson's $r=0.64, p<0.01$ ).

the infarct. We find that surviving L5 pyramidal cells in periinfarct cortex were equally affected, because there was no correlation between distance to the infarct edge and changes in length of dendritic tips or in the total dendritic arbor $(p=0.91)$ (supplemental Fig. 5, available at www.jneurosci.org as supplemental material). Similarly, there was no correlation between the size of the infarct and the changes in dendritic length (data not shown), perhaps because all the neurons we examined were located at least 1.3 away from the infarct edge. It is conceivable that, if we had imaged neurons right next to the infarct, a pruning versus distance relationship might have emerged.

\section{Discussion}

The role that surviving neurons play in the recovery of the lost functionalities after brain injury is still not well understood. Published studies using the Golgi method point to increased branching or elongation of dendrites in the contralateral hemisphere
(Jones and Schallert, 1992) as one possible strategy that pyramidal cells adopt to rewire the cortex. However, subsequent studies failed to replicate these results (Forgie et al., 1996; Prusky and Whishaw, 1996). Equally controversial are the data supporting large-scale dendritic plasticity of spared neurons in the area immediately surrounding a cortical lesion (Rowntree and Kolb, 1997; Gonzalez and Kolb, 2003), although this has been much less studied, especially after stroke. Such paradoxical results are perhaps not surprising considering that, with the absence of a pre-injury baseline in Golgi studies, investigators are forced to pool data from different groups of animals. This approach can probably only detect alterations in dendritic arbors of a very large magnitude. Golgi studies also failed to appreciate dynamic aspects of dendritic remodeling (e.g., simultaneous growth and pruning), which may be crucial for adaptive plasticity.

In addition, it is easy to be skeptical about the notion that dendrites of mature pyramidal neurons could grow to compensate for brain damage because they would have to overcome their natural tendency to remain stable, as revealed by in vivo imaging (Trachtenberg et al., 2002) (supplemental Fig. $4 a, b$, available at www. jneurosci.org as supplemental material). Thus, the motivation for this study was to settle this debate using a clinically relevant model of stroke and a chronic in vivo imaging approach that would allow us to monitor the same L5 pyramidal neurons, the main excitatory output cell of the neocortex, before and after a cortical lesion.

Using repeated imaging over 3 months, we saw no evidence of de novo branching or growth of apical dendritic trees in surviving L5 cells in peri-infarct cortex. On the contrary, we find that the complexity and length of apical arbors decrease slightly after stroke. These results are consistent with other studies that failed to demonstrate large-scale adaptive dendritic plasticity in peri-lesion cortex (Rowntree and Kolb, 1997; Gonzalez and Kolb, 2003).

However, our findings differ significantly from a recent in vivo two-photon microscopy study that examined the length and branching of pyramidal dendrites in peri-infarct cortex (Brown et al., 2010). The authors followed cortical pyramidal cells for up to 6 weeks after a photo-thrombotic stroke, which produces relatively tiny and well-circumscribed infarcts. The authors found evidence of dendritic branch tip growth away from the infarct in surviving neurons, but the sampling of neurons was biased toward a region immediately adjacent to the sharp edge of the Rose Bengal infarct. As a result, neurons were subjected to local tissue distortion, specifically ex vacuo pulling toward the infarct, and it is possible that the increase in dendritic branch tip length away from the infarct that they observed was simply attributable to stretching of the arbor (see below). 
In the present in vivo imaging study, we investigated largescale dendritic plasticity of $11 \mathrm{~L} 5$ pyramidal cells in peri-infarct cortex, over distances of up to $2.3 \mathrm{~mm}$ away from the infarct edge, for a period of 3 months, using the unilateral permanent MCAO model. This arterial occlusion stroke model produces much larger infarcts and is mechanistically similar to MCA strokes in humans. We find that dendritic pruning after stroke proceeds in a two-step process: first, there is a shortening of the dendritic arbor (including distal tips) during the first weeks, which is then followed by a slight loss of dendritic branches after the first month. It is conceivable that the two processes are mechanistically distinct and could have different triggers. For example, the delayed elimination of dendritic branches may selectively affect those that are either severely affected by ischemia or those that lost many of their axonal inputs (from cells that died in the infarct core).

Interestingly, both types of pruning were independent of the distance from the dendrite to the infarct edge $(1.3-2.3 \mathrm{~mm})$. This was somewhat surprising as we expected that dendrites closer to the infarct edge, because they were exposed to more ischemia, might suffer more retractions, as reported previously (Brown et al., 2010). The difference may reside in the fact that we sampled dendrites significantly farther away from the infarct edge.

An important finding of this paper is that the shorter length of dendrites after stroke reflected, at least in part, a general shrinkage of the entire apical arbor (not limited to dendritic tips). Our direct in vivo observations of significant warping of neuronal processes after stroke (Fig. 1b,c) suggest that this phenomenon is mainly attributable to tissue deformation around the infarct. Subsequent histological observations (supplemental Fig. 2, available at www.jneurosci.org as supplemental material) confirmed the severity of tissue distortion and shifts in neuronal structure. As a result, it is also likely that mechanical forces attributable to involution of the infarct after stroke account for a significant proportion of the slight branch tip elongations (e.g., from stretching) reported by Brown et al. (2010).

We conclude that L5 pyramidal cell apical dendrites in the peri-infarct cortex do not contribute to functional recovery after stroke through large-scale structural plasticity in the adult brain. However, we cannot rule out changes in the basilar dendritic tree of these cells or dendritic plasticity in other neuronal cell types (e.g., interneuron dendrites are known to be dynamic at baseline) (Lee et al., 2006). It is also important to mention that the absence of large-scale changes in dendritic complexity in peri-infarct cortex does not preclude the possibility of dendritic plasticity at finer spatial scales. For instance, changes in the density or turnover of dendritic spines, which have been documented in peri-infarct cortex, are likely to play a major role in circuit plasticity after stroke (Brown et al., 2009; Mostany et al., 2010). In addition, changes in spine turnover appear to also take place in the contralateral hemisphere (Takatsuru et al., 2009), suggesting that, even in the intact hemisphere, plasticity at the level of single synapses is more likely to contribute to functional recovery after stroke than large-scale remodeling of entire dendritic arbors. Future in vivo imaging studies should confirm whether rehabilitative strategies (Biernaskie and Corbett, 2001; Gibb et al., 2010) or manipulations of certain signaling pathways, such as PTEN (Chow et al., 2009) or fibroblast growth factor (Rowntree and Kolb, 1997), might induce dendritic remodeling after stroke.

\section{References}

Biernaskie J, Corbett D (2001) Enriched rehabilitative training promotes improved forelimb motor function and enhanced dendritic growth after focal ischemic injury. J Neurosci 21:5272-5280.
Biernaskie J, Chernenko G, Corbett D (2004) Efficacy of rehabilitative experience declines with time after focal ischemic brain injury. J Neurosci 24:1245-1254.

Brown CE, Aminoltejari K, Erb H, Winship IR, Murphy TH (2009) In vivo voltage-sensitive dye imaging in adult mice reveals that somatosensory maps lost to stroke are replaced over weeks by new structural and functional circuits with prolonged modes of activation within both the periinfarct zone and distant sites. J Neurosci 29:1719-1734.

Brown CE, Boyd JD, Murphy TH (2010) Longitudinal in vivo imaging reveals balanced and branch-specific remodeling of mature cortical pyramidal dendritic arbors after stroke. J Cereb Blood Flow Metab 30:783-791.

Carmichael ST (2008) Themes and strategies for studying the biology of stroke recovery in the poststroke epoch. Stroke 39:1380-1388.

Chow DK, Groszer M, Pribadi M, Machniki M, Carmichael ST, Liu X, Trachtenberg JT (2009) Laminar and compartmental regulation of dendritic growth in mature cortex. Nat Neurosci 12:116-118.

Dijkhuizen RM, Ren J, Mandeville JB, Wu O, Ozdag FM, Moskowitz MA, Rosen BR, Finklestein SP (2001) Functional magnetic resonance imaging of reorganization in rat brain after stroke. Proc Natl Acad Sci U S A 98:12766-12771.

Feng G, Mellor RH, Bernstein M, Keller-Peck C, Nguyen QT, Wallace M, Nerbonne JM, Lichtman JW, Sanes JR (2000) Imaging neuronal subsets in transgenic mice expressing multiple spectral variants of GFP. Neuron 28:41-51.

Forgie ML, Gibb R, Kolb B (1996) Unilateral lesions of the forelimb area of rat motor cortex: lack of evidence for use-dependent neural growth in the undamaged hemisphere. Brain Res 710:249-259.

Gibb RL, Gonzalez CL, Wegenast W, Kolb BE (2010) Tactile stimulation promotes motor recovery following cortical injury in adult rats. Behav Brain Res 214:102-107.

Gonzalez CL, Kolb B (2003) A comparison of different models of stroke on behaviour and brain morphology. Eur J Neurosci 18:1950-1962.

Holtmaat A, Bonhoeffer T, Chow DK, Chuckowree J, De Paola V, Hofer SB, Hübener M, Keck T, Knott G, Lee WC, Mostany R, Mrsic-Flogel TD, Nedivi E, Portera-Cailliau C, Svoboda K, Trachtenberg JT, Wilbrecht L (2009) Long-term, high-resolution imaging in the mouse neocortex through a chronic cranial window. Nat Protoc 4:1128-1144.

Jones TA, Schallert T (1992) Overgrowth and pruning of dendrites in adult rats recovering from neocortical damage. Brain Res 581:156-160.

Lee WC, Huang H, Feng G, Sanes JR, Brown EN, So PT, Nedivi E (2006) Dynamic remodeling of dendritic arbors in GABAergic interneurons of adult visual cortex. PLoS Biol 4:e29.

Marshall RS, Perera GM, Lazar RM, Krakauer JW, Constantine RC, DeLaPaz RL (2000) Evolution of cortical activation during recovery from corticospinal tract infarction. Stroke 31:656-661.

Mostany R, Portera-Cailliau C (2008) A craniotomy surgery procedure for chronic brain imaging. J Vis Exp pii:680.

Mostany R, Chowdhury TG, Johnston DG, Portonovo SA, Carmichael ST, Portera-Cailliau C (2010) Local hemodynamics dictate long-term dendritic plasticity in peri-infarct cortex. J Neurosci 30:14116-14126.

Pologruto TA, Sabatini BL, Svoboda K (2003) ScanImage: Flexible software for operating laser scanning microscopes. BioMed Eng OnLine 2:13.

Prusky G, Whishaw IQ (1996) Morphology of identified corticospinal cells in the rat following motor cortex injury: absence of use-dependent change. Brain Res 714:1-8.

Rowntree S, Kolb B (1997) Blockade of basic fibroblast growth factor retards recovery from motor cortex injury in rats. Eur J Neurosci 9:2432-2441.

Takatsuru Y, Fukumoto D, Yoshitomo M, Nemoto T, Tsukada H, Nabekura J (2009) Neuronal circuit remodeling in the contralateral cortical hemisphere during functional recovery from cerebral infarction. J Neurosci 29:10081-10086.

Tamura A, Graham DI, McCulloch J, Teasdale GM (1981) Focal cerebral ischaemia in the rat. 1. Description of technique and early neuropathological consequences following middle cerebral artery occlusion. J Cereb Blood Flow Metab 1:53-60.

Trachtenberg JT, Chen BE, Knott GW, Feng G, Sanes JR, Welker E, Svoboda $\mathrm{K}$ (2002) Long-term in vivo imaging of experience-dependent synaptic plasticity in adult cortex. Nature 420:788-794.

Zhang ZG, Chopp M (2009) Neurorestorative therapies for stroke: underlying mechanisms and translation to the clinic. Lancet Neurol 8:491-500. 